\title{
Problemas de comportamento e excesso de peso em pré-escolares do sul do Brasil
}

\author{
Behavior problems and overweight in preschool children in southern Brazil \\ Suélen Henriques da Cruz 1,2, Cesar Augusto Piccinini', Alícia Matijasevich³, Iná S. Santos²
}

\author{
Palavras-chave \\ Problemas de \\ comportamento, excesso de \\ peso, sobrepeso, obesidade, \\ pré-escolares.
}

\section{RESUMO}

Objetivo: Este estudo objetivou investigar a associação entre problemas de comportamento e excesso de peso (sobrepeso e obesidade) em pré-escolares de uma coorte de nascimentos do sul do Brasil, acompanhados do nascimento aos 4 anos de idade. Métodos: No acompanhamento dos quatro anos foram realizadas entrevistas domiciliares com as mães das crianças, tendo sido coletadas informações sobre a saúde e o desenvolvimento infantil, além de ser aplicado o Child Behavior Checklist 4-18/CBCL. Resultados: Cerca de 15\% dos meninos e 12\% das meninas de 4 anos apresentaram excesso de peso, e a associação entre problemas de comportamento e o excesso de peso nessa idade se deu apenas em problemas de ansiedade-depressão, nas meninas (OR: 3,79 [IC95\%: 1,60-8,97]; $p=0,002$ ). Conclusões: Meninas em idade pré-escolar com excesso de peso apresentaram mais problemas de ansiedade-depressão do que as com peso adequado da mesma idade. Essa informação é de extrema relevância, visto que os anos pré-escolares são ideais para se investir em prevenção, tanto dos problemas de comportamento, como do excesso de peso, possibilitando introduzir comportamentos/hábitos mais saudáveis e diminuir, assim, as consequências negativas que ambos acarretam à vida das crianças.

\begin{abstract}
Objective: This study aimed to investigate the association between behavior problems and excess of weight (overweight and obesity) in preschool children from a birth cohort in southern Brazil, followed from birth to four years old. Methods: In the follow-up of four years, home interviews with mothers were carried out where information on the health and development of children were collected, in addition to application of the Child Behavior Checklist 4-18/CBCL. Results: About 15\% of boys and 12\% of four-year girls were overweight/obese, and the association between behavior problems and excess of weight in this age occurred only with problems of anxiety-depression in girls (OR: 3.79 [95\% Cl: 1.60 to 8.97]; $p=0.002$ ). Conclusions: Preschool girls with excess of weight showed more problems of anxiety and depression than those with adequate weight at the same age. This information is extremely important, as the preschool years are ideal for investing in prevention of both behavioral problems and overweight/obesity, allowing to introduce healthier habits and behaviors and decrease thus the negative consequences both cause to children.
\end{abstract}

1 Universidade Federal do Rio Grande do Sul (UFRGS), Programa de Pós-Graduação em Psicologia. 2 Universidade Federal de Pelotas (UFPel), Programa de Pós-Graduação em Epidemiologia. 3 Universidade de São Paulo (USP), Escola de Medicina, Departamento de Medicina Preventiva.

Endereço para correspondência: Suélen Henriques da Cruz suepidemio@gmail.com 


\section{INTRODUÇÃO}

O excesso de peso (sobrepeso e obesidade) é considerado um sério desafio para a saúde pública mundial neste século, não só por suas altas prevalências, mas porque tem atingido também crianças e adolescentes, cada vez mais precocemente 1 . O excesso de peso na infância gera grande preocupação, pois, além de ser fator de risco para uma série de agravos à saúde física², também pode acarretar prejuízos a longo prazo, como obesidade na vida adulta e complicações que acompanham esse quadro ${ }^{3}$. Além disso, o excesso de peso também pode estar associado a dificuldades emocionais e sociais nas crianças, tais como autoestima mais baixa ${ }^{4-6}$, dificuldades de relacionamento ${ }^{7-9}$, sintomas de depressão e ansiedade ${ }^{4,5}$, além de menor qualidade de vida ${ }^{7,10}$. Por exemplo, em um estudo realizado na Austrália com 158 crianças de 7 a 11 anos de idade foi constatada associação negativa entre excesso de peso e autoestima, isto é, quanto maior o índice de massa corporal (IMC) das crianças, maior sua insatisfação corporal e, consequentemente, menor sua autoestima ${ }^{6}$. Em outro estudo, realizado por Braet et al. ${ }^{11}$ com uma amostra de 239 crianças belgas com idade entre nove e 12 anos, das quais 139 estavam obesas e 150 tinham peso adequado, foi constatado que as crianças obesas expressaram com maior frequência sentimentos negativos em relação ao autoconceito geral, isto é, não estavam apenas insatisfeitas com sua aparência pessoal, mas também com outros aspectos de sua vida.

Tendo em vista a relação entre excesso de peso e prejuízos à qualidade de vida das crianças em múltiplos domínios, especialmente na saúde mental, diversos estudos têm se dedicado a investigar a associação entre problemas de comportamento e excesso de peso em crianças e adolescentes $^{12-18}$. Por exemplo, em uma pesquisa realizada por Vila et al. ${ }^{13}$ com uma amostra clínica de crianças e adolescentes franceses de cinco a 17 anos de idade, crianças obesas apresentaram mais problemas de comportamento (entre 50\% e $64 \%$ da amostra) que seus controles, em especial problemas do tipo internalização. No estudo de Mustillo et al.18, os autores encontraram associação entre obesidade crônica com início na infância e continuidade na adolescência e problemas de externalização em meninos e meninas norte-americanos (idade entre nove e 16 anos). No estudo de Datar e Sturm ${ }^{15}$ com uma amostra de pré-escolares com 5 anos de idade, também norte-americanos, foi encontrada associação entre obesidade e problemas de externalização e de internalização em meninas, mas não em meninos.

Conforme pode ser visto, não há consenso na literatura em relação ao fato de as crianças com excesso de peso apresentarem mais problemas de comportamento do que aquelas com peso adequado. Essas divergências nos resultados podem estar associadas a questões metodológicas, entre elas a grande diversidade nas amostras estudadas, em particular em relação à idade das crianças, mas também quanto às características dos instrumentos utilizados para aferir problemas de comportamento. Somado a isso, muitas das amostras são clínicas, ou seja, compostas por crianças que estão buscando tratamento para o excesso de peso, o que as torna, em alguns aspectos, diferentes das crianças da população em geral ${ }^{11}$. Nota-se ainda que muitos estudos se dedicam a investigar crianças maiores e adolescentes, negligenciando o fato de que os anos pré-escolares são cruciais para o investimento em prevenção de problemas de saúde infantil $^{19}$, dentre os quais podem se destacar os problemas mentais e o excesso de peso. Sendo assim, a realização de um estudo de base populacional com o objetivo de investigar a associação entre problemas de comportamento e excesso de peso em pré-escolares é de suma importância para embasar estratégias de promoção do desenvolvimento infantil e também de intervenção nesse momento de vida tão propício a mudanças comportamentais que podem prevenir agravos na saúde das crianças. Com base no exposto, o presente estudo objetivou investigar a associação entre problemas de comportamento e excesso de peso em crianças pré-escolares de 4 anos, pertencentes a uma coorte de nascimentos do sul do Brasil.

\section{MÉTODOS}

\section{Participantes}

Participaram do estudo 3.750 crianças (51,8\% meninos) de 4 anos de idade e suas mães, oriundas de uma coorte de nascidos na zona urbana da cidade de Pelotas/RS em 2004. Durante o ano de 2004, 4.263 bebês nascidos vivos foram identificados e suas mães foram convidadas a fazer parte do estudo. Foram realizadas entrevistas aos três, 12 e 24 meses de vida das crianças, bem como aos 4 anos de idade, tendo sido coletados, nessas ocasiões, diversos dados sobre saúde e desenvolvimento infantil. A história e a metodologia deste estudo foram descritas por Santos et al..$^{20}$ A Tabela 1 apresenta uma descrição dos participantes da coorte. Conforme pode ser visto, cerca de 15\% das crianças nasceram pré-termo e $10 \%$ com baixo peso; a maioria era branca $(67,6 \%)$; e não tinha irmãos $(78,6 \%)$. Com relação às mães, quase metade da amostra (49,5\%) tinha idade entre 20 e 29 anos no nascimento das crianças; a maioria possuía oito anos completos ou mais de estudo; 79,7\% das mães tinham companheiro residindo na mesma casa. Não houve diferença entre os sexos em relação às variáveis descritas anteriormente.

\section{Delineamento e procedimento}

O presente estudo faz parte de um estudo epidemiológico maior, longitudinal, cujo objetivo geral é investigar/acompanhar os determinantes de saúde e doença em uma coorte de 
Tabela 1. Características da amostra aos 4 anos, por sexo das crianças

\begin{tabular}{|c|c|c|c|}
\hline \multirow{2}{*}{ Variáveis } & Total & Meninos & Meninas \\
\hline & $\%(\mathrm{~N})$ & $\%(\mathrm{~N})$ & $\%(\mathrm{~N})$ \\
\hline \multicolumn{4}{|l|}{ Criança } \\
\hline \multicolumn{4}{|c|}{ Idade gestacional $(\mathrm{n}=3.746)$} \\
\hline$<37$ & $13,7(515)$ & $13,4(260)$ & $14,1(255)$ \\
\hline$\geq 37$ & $86,3(3.231))$ & $86,6(1.682)$ & $85,9(1.549)$ \\
\hline \multicolumn{4}{|c|}{ Peso ao nascer ( $n=3.749)$} \\
\hline$<2.500 \mathrm{~g}$ & 9,3(347) & $8,1(157)$ & $10,5(190)$ \\
\hline$\geq 2.500 \mathrm{~g}$ & $90,7(3.402)$ & $91,9(1.787)$ & $89,5(1.615)$ \\
\hline \multicolumn{4}{|c|}{ Cor da pele $(n=3.534)$} \\
\hline Branca & $67,7(2.391)$ & $67,6(1.240)$ & $67,7(1.151)$ \\
\hline Não branca & $32,3(1.143)$ & $32,4(594)$ & $32,3(549)$ \\
\hline \multicolumn{4}{|c|}{ Irmãos mais novos $(\mathrm{n}=3.750)$} \\
\hline Nenhum & $78,7(2.951)$ & $78,7(1.530)$ & $78,7(1.421)$ \\
\hline 1 & $19,4(726)$ & $19,3(376)$ & $19,4(350)$ \\
\hline 2 ou mais & $1,9(73)$ & $2,0(38)$ & $1,9(35)$ \\
\hline \multicolumn{4}{|l|}{ Mãe } \\
\hline \multicolumn{4}{|l|}{ Idade $(\mathrm{n}=3.667)$} \\
\hline$<20$ & $18,7(687)$ & $18,4(347)$ & $19,1(340)$ \\
\hline $20-29$ & $49,6(1.818)$ & $50,4(953)$ & $48,7(865)$ \\
\hline $30-39$ & $28,3(1.037)$ & $27,9(527)$ & $28,7(510)$ \\
\hline $40+$ & $3,4(125)$ & $3,3(63)$ & $3,5(62)$ \\
\hline \multicolumn{4}{|c|}{ Tem marido/companheiro $(n=3.742)$} \\
\hline Não & $20,2(754)$ & $20,0(388)$ & $20,3(366)$ \\
\hline Sim & $79,8(2.988)$ & $80,0(1.551)$ & $79,7(1.437)$ \\
\hline \multicolumn{4}{|c|}{ Escolaridade $(n=3.735)$} \\
\hline $0-3$ & $7,7(287)$ & $7,4(143)$ & $8,0(144)$ \\
\hline $4-7$ & $32,0(1.195)$ & $31,7(614)$ & $32,3(581)$ \\
\hline $8-10$ & $22,3(834)$ & $21,4(413)$ & $23,3(421)$ \\
\hline $11+$ & $38,0(1.419)$ & $39,5(764)$ & $36,4(655)$ \\
\hline \multicolumn{4}{|c|}{ Índice de bens ( $\mathrm{n}=3.748)$} \\
\hline Q1 (mais baixo) & $10,5(394)$ & $10,5(204)$ & $10,5(190)$ \\
\hline Q2 & $15,2(571)$ & $13,7(267)$ & $16,8(304)$ \\
\hline Q3 & $23,2(869)$ & $23,0(447)$ & $23,4(422)$ \\
\hline Q4 & $20,5(766)$ & $21,3(413)$ & $19,6(353)$ \\
\hline Q5 (mais alto) & $30,6(1.148)$ & $31,5(612)$ & $29,7(536)$ \\
\hline
\end{tabular}

crianças. O delineamento do presente estudo é transversal, e seu objetivo específico é investigar a associação entre problemas de comportamento e excesso de peso nas crianças da coorte aos 4 anos de idade. As variáveis utilizadas foram obtidas em diferentes momentos, tais como por ocasião do nascimento das crianças (idade gestacional e peso ao nascer) e aos 4 anos de idade (cor da pele e número de irmãos mais novos). As variáveis idade gestacional, peso ao nascer e número de irmãos mais novos foram coletadas de forma contínua, sendo posteriormente categorizadas. A variável cor da pele foi referida pela mãe da criança, sendo após categorizada em branca e não branca (incluindo nesta categoria preta, mulata, amarela e outra). Dados maternos foram obtidos nos acompanhamentos perinatal (idade materna no nascimento da criança) e dos quatro anos (escolaridade, presença de companheiro, índice de bens familiares). A variável idade materna foi calculada com base nas datas de nascimento da mãe e na data da entrevista e posteriormente categorizada; a escolaridade materna foi coletada de forma contínua como anos completos de estudo e categorizada em quatro categorias; para a variável presença de companheiro foi considerado se a mãe tinha um companheiro residindo na mesma casa que ela e a criança; o índice de bens familiares (IEN) foi obtido com o preenchimento de um questionário sobre os bem materiais presentes no domicílio, sendo posteriormente dividido em quintis tendo como referência os valores de IEN para a cidade Pelotas: 0; 281, 368, 476, 619 e 2000.

Para a obtenção da prevalência de problemas de comportamento nas crianças, foi aplicado aos quatro anos o Child Behavior Checklist 4-18 (CBCL) $)^{21}$. O CBCL é um instrumento padronizado e validado que possui 118 itens, os quais descrevem comportamentos infantis referentes a sintomas psicopatológicos investigados em oito escalas: I) retraimento; II) queixas somáticas; III) ansiedade/depressão; IV) problemas com contato social; V) problemas de pensamento; VI) problemas de atenção; VII) comportamento de quebrar regras/disruptivo; e VIII) comportamento agressivo. Devido à variação no nível de escolaridade das mães das crianças no presente estudo, ele era lido pelas entrevistadoras e as mães faziam suas escolhas a cada item, classificando se o comportamento descrito era: ausente (escore $=0$ ); às vezes presente (escore $=1$ ); ou frequentemente presente (escore $=2$ ). O total de problemas de comportamento foi calculado a partir da soma dos escores brutos obtidos em todas as escalas. Os problemas de internalização foram obtidos pela soma do total de pontos das escalas I, II e III, enquanto os problemas de externalização, pela soma dos pontos das escalas VII e VIII. O CBCL foi adaptado e validado no Brasil por Bordin et al.22 e os dados deste estudo para a população brasileira mostraram um bom nível de sensibilidade $(87,0 \%$ a $75,0 \%$ para casos leves, 95,4\% para moderados e 100\% para severos) usando o ponto de corte para a população norte-americana, ainda que não tenha sido possível medir especificidade devido ao pequeno número de crianças saudáveis contido na amostra. Pelo fato de não ter sido realizado um estudo em nível populacional, o ponto de corte usado para a obtenção de escore-T foi o mesmo derivado da população norte-americana. Entretanto, por ser um instrumento amplamente utilizado e validado em diversos países, a aplicação do CBCL no presente estudo justifica-se também pela validade multicultural do instrumento ${ }^{23}$. Os escores brutos do CBCL foram transformados em escores padronizados de acordo com a pontuação da amostra normativa (escore T) e, a partir da padronização dos escores, as crianças foram classificadas como não clínicas (escores $T<67$ ), limítrofes $(T \geq 67$ e $\leq 70$ ) e clínicas ( $T>70$ ), para cada escala do CBCL/4-18. Em relação à soma das escalas (internalização e externalização), as crianças foram consideradas como não clínicas $(T<60)$, limítrofes $(T \geq 60$ e $\leq 63)$ e clínicas ( $T>63)$. Optou-se por incluir as crianças limítrofes na categoria clínica, conforme sugerido por Achembach ${ }^{21}$ em situações de pesquisa. Cabe 
ressaltar que uma mesma criança pode ser classificada como clínica em diferentes escalas.

O peso e a altura das crianças foram coletados aos quatro anos, aferidos por meio de balanças e estadiômetros de alta precisão. Foram consideradas com peso normal as crianças que apresentavam um escore- $z$ de IMC para a idade entre -2 e +2 desvios-padrão (percentil de 3 a 97), ou seja, foram incluídas nesse grupo as crianças classificadas segundo a Organização Mundial da Saúde 1 como eutróficas e em risco de desenvolver sobrepeso. Foram consideradas com excesso de peso as crianças com escore-z de IMC para idade igual ou superior a +2 , isto é, incluídas nessa categoria as crianças tanto com sobrepeso como com obesidade.

Os dados coletados foram registrados no software EPI-INFO versão 6.04, com checagem automática de amplitude e consistência. Para a análise do banco de dados foi utilizado o pacote estatístico STATA 14.0. Foram descritas as frequências absolutas e relativas de todas as variáveis estudadas, sendo calculada também a prevalência de excesso de peso de acordo com a exposição a cada uma das variáveis. Após, foram conduzidas análises de regressão logística bruta e ajustada, tendo o excesso de peso como desfecho dicotômico e as variáveis problemas de comportamento como exposições de interesse. Para a análise ajustada foram consideradas como potenciais fatores de confusão as variáveis maternas (idade, escolaridade, ter marido/companheiro, e renda familiar) e da criança (idade gestacional, peso ao nascer, cor da pele e número de irmãos). Todas as análises foram conduzidas com estratificação por sexo das crianças, pelo fato de o IMC variar conforme o sexo, pois para seu cálculo se consideram as curvas de crescimento de meninos e meninas, que são diferentes.

O projeto de pesquisa envolvendo a Coorte de 2004, do qual o presente estudo faz parte, foi aprovado pelo Comitê de Ética em Pesquisa da Faculdade de Medicina da Universidade Federal de Pelotas, tendo sido considerado adequado do ponto de vista ético e metodológico, de acordo com as Diretrizes e Normas Regulamentadoras de Pesquisa envolvendo Seres Humanos (Resoluções 196/96 e 466/2012 do Conselho Nacional de Saúde). Desde o início do estudo, os participantes foram informados sobre os objetivos e os procedimentos da pesquisa e puderam decidir livremente sobre sua participação, sendo-lhes assegurada, dessa forma, sua autonomia. A privacidade e a confidencialidade das informações foram resguardadas e todos os participantes do estudo assinaram um termo de consentimento livre e esclarecido.

\section{RESULTADOS}

A prevalência de excesso de peso aos 4 anos foi de 15,1\% ( $n=259)$ nos meninos [sobrepeso 9,1\% ( $n=157) ;$ obesidade $5,9 \%(n=102)$ ] e de $11,9 \%(n=190)$ nas meninas [sobrepeso
$7,6 \%(n=121) ;$ obesidade $4,3 \%(n=69)]$. No que se refere à ocorrência de problemas de comportamento e sua associação com excesso de peso, as Tabelas 2 e 3 apresentam a descrição dos resultados e as associações bruta e ajustada entre os meninos e as meninas, respectivamente. Conforme pode ser visto na Tabela 2, entre os meninos, 33,6\% ( $n=653)$ apresentaram problemas de comportamento (escore total do CBCL classificado como clínico), 19,1\% ( $n=372)$ deles apresentaram problemas do tipo internalização e 40,6\% ( $n=790)$ problemas de externalização. Os problemas de comportamento mais prevalentes foram agressividade, que ocorreu em 17,6\% ( $n=342)$ dos meninos, e comportamento de quebrar regras, cuja prevalência foi de 17,4\% ( $n=338)$. Pode-se observar que, entre os meninos que apresentaram problemas de comportamento, $14,6 \%(n=95)$ deles tinham excesso de peso, ao passo que $15,2 \%(n=196)$ dos que não apresentavam problemas de comportamento possuíam excesso de peso. Não houve associação significativa entre nenhum dos problemas de comportamento investigados e o excesso de peso entre os meninos, conforme pode ser visto pelos odds ratios (OR) e seus intervalos de confiança de $95 \%$ (IC95\%) (Tabela 2).

Entre as meninas, $37,7 \%(n=681)$ apresentaram problemas de comportamento, $11,6 \%(n=210)$ delas manifestaram problemas de internalização e 48,3\% ( $n=873)$, de externalização (Tabela 3). Assim como nos meninos, os problemas de comportamento de agressividade e de quebrar regras também foram os mais prevalentes entre as meninas, ocorrendo em $23,8 \%(n=430)$ e $23,9 \%(n=431)$ delas, respectivamente. Dentre as meninas que apresentavam problemas de comportamento, 11,7\% ( $n=80)$ também tinham excesso de peso, enquanto $12,0 \%(n=135)$ das que não apresentavam problemas de comportamento tinham excesso de peso. Entre as meninas, os problemas de ansiedade-depressão já na análise bruta mostraram associação significativa com excesso de peso, que se tornou ainda mais forte quando realizado o controle para as variáveis sociodemográficas. Pode-se observar, assim, que as meninas que apresentaram problemas de ansiedade-depressão tiveram chance maior de apresentar também excesso de peso, quando comparadas às demais (OR: 3,79 [IC95\%: 1,60-8,97]; $p=0,002$ ). Não houve associação entre os demais problemas de comportamento e o excesso de peso entre as meninas.

\section{DISCUSSÃO}

Apesar de não ter sido encontrada associação entre problemas de comportamento e excesso de peso no presente estudo, resultado que está de acordo com outros estudos com pré-escolares ${ }^{24,25}$, foi verificada associação específica entre problemas de ansiedade-depressão nas meninas. Esse achado apoia o do estudo de Datar e Sturm ${ }^{15}$, que também 
Tabela 2. Descrição dos problemas de comportamento e sua associação com excesso de peso aos 4 anos entre os meninos $(n=1.944)$

\begin{tabular}{|c|c|c|c|c|c|c|}
\hline \multirow{2}{*}{ Variáveis } & \multirow{2}{*}{$\%(\mathrm{~N})$} & \multirow{2}{*}{ \% Excesso de peso } & \multirow[b]{2}{*}{$O R\left(I_{956}\right)$} & \multirow[b]{2}{*}{$p$} & \multicolumn{2}{|c|}{ Análise ajustada* } \\
\hline & & & & & $O R\left(I_{95 \%}\right)$ & $p$ \\
\hline Escore total & & & & 0,760 & & 0,877 \\
\hline Não & 66,4 (1.291) & 15,2 (196) & 1,00 & & 1,00 & \\
\hline Sim & $33,6(653)$ & $14,6(95)$ & $0,96(0,72-1,27)$ & & $0,98(0,72-1,32)$ & \\
\hline Internalização & & & & 0,704 & & 0,914 \\
\hline Não & $80,9(1.572)$ & $15,2(239)$ & 1,00 & & 1,00 & \\
\hline Sim & $19,1(372)$ & $14,3(53)$ & $0,94(0,66-1,32)$ & & $0,98(0,68-1,42)$ & \\
\hline Externalização & & & & 0,243 & & 0,372 \\
\hline Não & $59,4(1.154)$ & $15,9(183)$ & 1,00 & & 1,00 & \\
\hline Sim & $40,6(790)$ & $13,8(109)$ & $0,85(0,64-1,12)$ & & $0,87(0,65-1,17)$ & \\
\hline Retraimento & & & & 0,489 & & 0,551 \\
\hline Não & $93,2(1.812)$ & $15,2(275)$ & 1,00 & & 1,00 & \\
\hline Sim & $6,8(132)$ & $12,7(17)$ & $0,82(0,46-1,45)$ & & $0,83(0,44-1,55)$ & \\
\hline Queixas somáticas & & & & 0,742 & & 0,845 \\
\hline Não & $95,8(1.863)$ & $15,0(279)$ & 1,00 & & 1,00 & \\
\hline Sim & $4,2(81)$ & $16,4(13)$ & $1,12(0,58-2,16)$ & & $1,07(0,53-2,15)$ & \\
\hline Contato social & & & & 0,478 & & 0,562 \\
\hline Não & $96,7(1.880)$ & $15,1(284)$ & 1,00 & & 1,00 & \\
\hline Sim & $3,3(64)$ & $11,5(7)$ & $0,73(0,31-1,73)$ & & $0,76(0,29-1,95)$ & \\
\hline Ansiedade/depressão & & & & 0,404 & & 0,543 \\
\hline Não & $97,5(1.896)$ & $15,1(286)$ & 1,00 & & 1,00 & \\
\hline Sim & $2,5(48)$ & $10,3(5)$ & $0,64(0,23-1,82)$ & & $0,72(0,25-2,07)$ & \\
\hline Problemas de atenção & & & & 0,407 & & 0,902 \\
\hline Não & $97,9(1.903)$ & $14,9(283)$ & 1,00 & & 1,00 & \\
\hline Sim & $2,1(40)$ & $20,0(8)$ & $1,46(0,62-3,30)$ & & $0,93(0,32-2,75)$ & \\
\hline Agressividade & & & & 0,240 & & 0,278 \\
\hline Não & $82,4(1.602)$ & $15,5(248)$ & 1,00 & & 1,00 & \\
\hline Sim & $17,6(342)$ & $12,8(44)$ & $0,80(0,55-1,16)$ & & $0,81(0,54-1,19)$ & \\
\hline Comportamento de quebrar regras & & & & 0,138 & & 0,220 \\
\hline Não & $82,6(1.606)$ & $15,6(250)$ & 1,00 & & 1,00 & \\
\hline Sim & $17,4(338)$ & $12,2(41)$ & $0,75(0,52-1,09)$ & & $0,76(0,52-1,16)$ & \\
\hline Problemas de pensamento & & & & 0,160 & & 0,373 \\
\hline Não & $94,8(1.843)$ & $14,7(271)$ & 1,00 & & 1,00 & \\
\hline Sim & $5,2(101)$ & $15,2(15)$ & $1,47(0,86-2,51)$ & & $1,31(0,72-2,40)$ & \\
\hline
\end{tabular}

* Ajuste para: idade e escolaridade maternas, presença de companheiro, índice de bens familiares, idade gestacional, peso ao nascer, cor da pele e número de irmãos.

revelou associação entre problemas de ansiedade-depressão e excesso de peso em meninas norte-americanas em idade pré-escolar (4 a 5 anos). De forma geral, a associação entre problemas de ansiedade-depressão e excesso de peso tem sido encontrada em meninas maiores ${ }^{26-28}$. No estudo de Erickson et al. ${ }^{28}$, por exemplo, que investigaram pré-adolescentes norte-americanos, sintomas depressivos nas meninas estiveram mais fortemente associados à preocupação com o excesso de peso corporal, que pode ser reforçada pelo estigma social de que são vítimas as crianças com sobrepeso/ obesidade, desde muito cedo, tanto por parte de pares ${ }^{29,30}$, como de professores ${ }^{31}$ e até mesmo dos pais ${ }^{32}$.

Esses achados da literatura, junto com os revelados no presente estudo, apontam para a possibilidade de crianças em idade pré-escolar, em especial meninas com excesso de peso, já sofrerem de problemas de ansiedade-depressão associados à sua autoimagem. De fato, o estudo pioneiro de Cramer e Steinwert ${ }^{33}$ realizado com pré-escolares revelou que já são encontrados sinais de estigmatização do excesso de peso em crianças norte-americanas aos 3 anos de idade. 
Tabela 3. Descrição dos problemas de comportamento e sua associação com excesso de peso aos 4 anos entre as meninas ( $\mathrm{n}=1.806)$

\begin{tabular}{|c|c|c|c|c|c|c|}
\hline \multirow{2}{*}{ Variáveis } & \multirow{2}{*}{$\%(\mathrm{~N})$} & \multirow{2}{*}{ \%Excesso de peso } & \multicolumn{2}{|c|}{ Análise bruta } & \multicolumn{2}{|c|}{ Análise ajustada* } \\
\hline & & & $O R\left(I C_{95 \%}\right)$ & $p$ & $O R\left(I_{95 \%}\right)$ & $p$ \\
\hline Escore total & & & & 0,841 & & 0,536 \\
\hline Não & $62,3(1.125)$ & $12,0(135)$ & 1,00 & & 1,00 & \\
\hline Sim & $37,7(681)$ & $11,7(80)$ & $0,97(0,71-1,33)$ & & $1,11(0,79-1,56)$ & \\
\hline Internalização & & & & 0,530 & & 0,100 \\
\hline Não & $88,4(1.596)$ & $11,7(187)$ & 1,00 & & 1,00 & \\
\hline Sim & $11,6(210)$ & $13,3(28)$ & $1,16(0,73-1,83)$ & & $1,49(0,93-2,40)$ & \\
\hline Externalização & & & & 0,691 & & 0,723 \\
\hline Não & $51,7(933)$ & $12,2(114)$ & 1,00 & & 1,00 & \\
\hline Sim & $48,3(873)$ & $11,6(101)$ & $0,94(0,69-1,28)$ & & $1,06(0,77-1,47)$ & \\
\hline Retraimento & & & & 0,483 & & 0,201 \\
\hline Não & $96,7(1.746)$ & $11,8(206)$ & 1,00 & & 1,00 & \\
\hline Sim & $3,3(60)$ & $15,2(91)$ & $1,34(0,59-3,04)$ & & $1,73(0,75-4,01)$ & \\
\hline Queixas somáticas & & & & 0,699 & & 0,933 \\
\hline Não & $97,8(1.767)$ & $12,0(212)$ & 1,00 & & 1,00 & \\
\hline Sim & $2,2(39)$ & $9,7(4)$ & $0,79(0,24-2,62)$ & & $0,95(0,28-3,21)$ & \\
\hline Contato social & & & & 0,560 & & 0,988 \\
\hline Não & $97,1(1.753)$ & $12,0(210)$ & 1,00 & & 1,00 & \\
\hline Sim & $2,9(53)$ & $9,1(5)$ & $0,73(0,26-2,08)$ & & $0,99(0,34-2,88)$ & \\
\hline Ansiedade/depressão & & & & 0,020 & & 0,002 \\
\hline Não & $97,8(1.767)$ & $11,6(205)$ & 1,00 & & 1,00 & \\
\hline Sim & $2,2(39)$ & $25,8(10)$ & $2,64(1,16-6,00)$ & & $3,79(1,60-8,97)$ & \\
\hline Problemas de atenção & & & & 0,253 & & 0,451 \\
\hline Não & $96,3(1.740)$ & $11,1(193)$ & 1,00 & & 1,00 & \\
\hline Sim & $3,7(66)$ & $7,0(5)$ & $0,54(0,19-1,53)$ & & $0,70(0,24-1,90)$ & \\
\hline Agressividade & & & & 0,771 & & 0,249 \\
\hline Não & $76,2(1.376)$ & $11,8(162)$ & 1,00 & & 1,00 & \\
\hline Sim & $23,8(430)$ & $12,3(53)$ & $1,05(0,74-1,50)$ & & $1,24(0,86-1,83)$ & \\
\hline Comportamento de quebrar regras & & & & 0,754 & & 0,205 \\
\hline Não & $76,1(1.375)$ & $11,8(162)$ & 1,00 & & 1,00 & \\
\hline Sim & $23,9(431)$ & $12,4(53)$ & $1,06(0,74-1,51)$ & & $1,27(0,87-1,85)$ & \\
\hline Problemas de pensamento & & & & 0,538 & & 0,903 \\
\hline Não & $94,8(1.713)$ & $12,0(205)$ & 1,00 & & 1,00 & \\
\hline Sim & $5,2(93)$ & $9,8(91)$ & $0,79(0,38-1,67)$ & & $0,95(0,45-2,03)$ & \\
\hline
\end{tabular}

* Ajuste para: idade e escolaridade maternas, presença de companheiro, índice de bens familiares, idade gestacional, peso ao nascer, cor da pele e número de irmãos.

Nesse estudo, as crianças tenderam a atribuir mais características negativas a figuras com excesso de peso do que a figuras com peso normal. Além disso, tais crianças mencionaram preferir brincar com colegas de peso normal e não com os que apresentavam excesso de peso. Especificamente no que diz respeito à associação entre forma física e diferenças de gênero em pré-escolares, um estudo com crianças inglesas de 2 a 5 anos de idade indicou que as figuras de meninas com sobrepeso são julgadas mais negativamente pelas crianças do que as de meninos ${ }^{34}$. Nesse sentido, pode-se pensar que o fato de as meninas sofrerem mais dos estigmas sociais ligados à forma física advém não só dos padrões culturais de beleza contemporâneos, em que as mulheres precisam ser magras e esguias, mas também do convívio com os próprios pais, que reforçam esses padrões no contexto familiar proporcionando às crianças uma forma negativa e estigmatizada de ver as pessoas com peso excessivo, desde pequenas. Um exemplo disso é apontado pelo estudo de Davison e Birch ${ }^{32}$, que, ao investigarem estereótipos em meninas de 9 anos de idade e em seus pais, encontraram que ambos atribuíam características negativas a pessoas com excesso de peso, em comparação a pessoas 
mais magras. Segundo esse estudo, pais e mães com maior nível educacional e renda, assim como aqueles que relatavam grande investimento em sua aparência, eram os que mais utilizavam estereótipos em relação ao excesso de peso. Nessa perspectiva, os pais podem estar contribuindo para o sofrimento psicológico das crianças com excesso de peso, ao estigmatizarem eles próprios os filhos (em especial as fiIhas). Por exemplo, o fato de os pais se sentirem culpados e criticados pelo excesso de peso dos filhos, apontado no estudo de Pierce e Wardle ${ }^{35}$, juntamente com o sentimento de frustração por não conseguirem ajudar suas crianças a perderem peso, pode fazer com que adotem atitudes e comportamentos estigmatizados, tais como criticar e fazer comentários negativos a respeito da aparência.

Assim sendo, pode-se pensar na possibilidade de o excesso de peso ocasionar consequências psicológicas negativas, especialmente em meninas bem pequenas, que podem apresentar comportamentos de ansiedade-depressão devido ao estigma social do qual são vítimas, não só no meio social mais amplo, mas na própria família. Além de problemas de ansiedade-depressão, encontrados no presente estudo, é possível que as meninas com excesso de peso também apresentem dificuldades sociais, ligadas aos estigmas dos quais são alvo, como revelado pelo estudo de Griffiths et al. ${ }^{36}$. Ao investigar problemas de comportamento em crianças de 3 a 5 anos com excesso de peso, os autores encontraram que, aos 3 anos de idade, as meninas com sobrepeso/obesidade apresentavam mais problemas sociais do que meninas com peso adequado.

Conforme destacado anteriormente, os resultados do presente estudo revelaram uma associação entre problemas de comportamento e excesso de peso já aos 4 anos de idade, ainda que parcial, pois essa associação somente foi encontrada em meninas. Entretanto, tal associação não garante que o excesso de peso seja preditor dos problemas de comportamento, ou vice-versa, tendo em vista que não é possível apontar a direcionalidade desses achados. Segundo Garthus-Niegel et al.24, há evidências de uma inter-relação entre os padrões cerebrais responsáveis pelo apetite e pela regulação emocional e, em virtude disso, um conjunto comum de fatores pode contribuir tanto para a manifestação de problemas de comportamento quanto para o risco de excesso de peso. Assim, de acordo com esses autores, esses mecanismos não se excluem mutuamente, sendo possível uma relação recíproca entre problemas de comportamento e peso ao longo do tempo, de forma que esses problemas acarretem excesso de peso e o excesso de peso também venha a acarretar problemas de comportamento.

No que se refere às limitações do presente estudo, alguns aspectos merecem ser destacados. Primeiramente, salienta-se a falta de poder para estudar as associações com os desfechos de menor prevalência. Além disso, o fato de as informações sobre os problemas de comportamento nas crianças terem sido obtidas apenas com base nos relatos das mães pode ter levado a uma subestimação dos problemas de comportamento, visto que, ao estarem emocionalmente envolvidas com as crianças, as mães podem ter dificuldade de detectar a ocorrência de problemas nelas. Somado a isso, pelo fato de a identificação dos problemas comportamentais nas crianças depender também do papel que os adultos desempenham em suas vidas, muitos problemas podem não ser detectados até mesmo pelos pais, sugerindo que a ocorrência de problemas de comportamento pode ser subestimada, particularmente os de internalização ${ }^{37,38}$, que costumam ser mais difíceis de serem notados. Seria mais adequado, assim, acessar os problemas de comportamento na infância por meio de outros informantes (por exemplo, professores), já que existe a possibilidade de o relato dos pais refletir o estado mental deles próprios ou a relação pais-criança em adição aos sintomas inerentes à criança ${ }^{39,40}$. Cabe salientar ainda que, ao se interpretar os resultados do presente estudo, deve-se levar em consideração que os problemas de comportamento das crianças foram acessados apenas por meio de um instrumento que, além disso, é de rastreamento. $\mathrm{O}$ ideal para avaliar a presença de problemas de saúde mental nas crianças seria utilizar uma combinação de instrumentos, bem como entrevistas clínicas, entretanto, dada a natureza epidemiológica deste estudo, abarcando um número grande de sujeitos ( $n=3.750$ ), o instrumento utilizado oferece informações úteis, apesar de não oferecer especificidade em termos diagnósticos.

Apesar das referidas limitações, entretanto, acredita-se que o presente estudo traz contribuições importantes ao investigar a associação entre problemas de comportamento e o excesso de peso em uma amostra populacional de crianças pré-escolares. O fato de meninas com excesso de peso apresentarem mais problemas de ansiedade-depressão do que seus pares com peso adequado, sugere a presença de sofrimento psicológico ao qual estão expostas essas crianças com excesso de peso. Nesse sentido, acredita-se ser importante tanto ações de prevenção do excesso de peso infantil, problema este que pode ocasionar diversos prejuízos à saúde das crianças, como também uma maior conscientização social sobre o fato de crianças com excesso de peso estarem mais sujeitas a preconceito e estigmatização, que podem ocasionar consequências psicológicas negativas, tais como os problemas de comportamento.

Deve-se levar em consideração, ainda, que os anos pré-escolares são ideais para se investir em prevenção, tanto dos problemas de comportamento, como do excesso de peso, introduzindo comportamentos/hábitos mais saudáveis às crianças, bem como intervindo em questões familiares e parentais associadas ao excesso de peso. Sabe-se, pois, que fatores como a estrutura e o contexto familiar ${ }^{41,42}$, além de psicopatologia parental (como depressão e ansiedade maternas, por exemplo) ${ }^{43}$, exercem importante papel na gênese 
tanto dos problemas de comportamento quanto do excesso de peso nas crianças, especialmente entre as de menor idade.

\section{CONCLUSÃO}

Foi encontrada associação entre problemas de ansiedade-depressão em meninas com excesso de peso aos 4 anos de idade, indicando que meninas com excesso de peso, comparadas às com peso adequado, apresentam mais problemas de comportamento de ansiedade-depressão.

\section{CONTRIBUIÇÕES INDIVIDUAIS}

Suélen Henriques da Cruz - Participou da concepção e do desenho do estudo, da revisão de literatura, análise e interpretação dos dados e da elaboração do artigo e aprovação da versão final.

Cesar Augusto Piccinini - Participou da concepção do desenho do estudo, da análise e interpretação dos dados, da revisão crítica do conteúdo intelectual do artigo e aprovação da versão final.

Iná S. Santos - Pesquisadora coordenadora do Estudo da Coorte de 2004. Participou da concepção do desenho do estudo, da interpretação dos dados, da revisão crítica do conteúdo intelectual do artigo e aprovação da versão final.

Alícia Matijasevich - Pesquisadora coordenadora do Estudo da Coorte de 2004. Participou da concepção do desenho do estudo, da revisão crítica do conteúdo intelectual do artigo e aprovação da versão final.

\section{CONFLITOS DE INTERESSE}

Os autores declaram, individualmente, não haver quaisquer conflitos de interesse na publicação do presente manuscrito.

\section{AGRADECIMENTOS}

Agradecemos a Wellcome Trust pelo financiamento do estudo da Coorte de Nascimentos de 2004, bem como ao apoio às fases anteriores do estudo, fornecido pelo pela Organização Mundial da Saúde, Programa Nacional de Apoio a Núcleos de Excelência (PRONEX), Conselho Nacional de Pesquisa (CNPq), Ministério da Saúde e Pastoral da Criança.

\section{REFERÊNCIAS}

1. World Health Organization - The WHO Child Growth Standards. Disponível em: <http:// www.who.int/childgrowth/en/>. Acesso em: 20 mar. 2012.
2. World Health Organization - Global Strategy on Diet, Physical Activity and Health Disponível em: <http://www.who.int/dietphysicalactivity/childhood_schools/en/index. html>. Acesso em: 31 mar. 2012.

3. Gurnani M, Birken C, Hamilton J. Childhood Obesity: Causes, Consequences, and Management. Pediatr Clin North Am. 2015;10(3):234-44.

4. Pulgarón ER. Childhood obesity: a review of increased risk for physical and psychological comorbidities. Clin Ther. 2013;35(1):18-32.

5. Russell-Mayhew S, McVey G, Bardick A, Ireland A. Mental health, wellness, and childhood overweight/obesity. J Obes. 2012;2012:281801.

6. Taylor A, Wilson C, Slater A, Mohr P. Self-esteem and body dissatisfaction in young children: Associations with weight and perceived parenting style. Clin Psych. 2012;16(1):25-35.

7. Lee PY, Cheah WL, Chang CT, Siti RG. Childhood obesity, self-esteem and health-related quality of life among urban primary schools children in Kuching, Sarawak, Malaysia. Malays J Nutr. 2012;18(2):207-19.

8. Pitrou I, Shojaei T, Wazana A, Gilbert F, Kovess-Masféty V. Child overweight, associated psychopatology, and social functioning: a french school-based survey in 6- to 11-year-old children. Obesity. 2010;18(4):809-17.

9. Griffiths LJ, Parsons TJ, Hill AJ. Self-esteem and quality of life in obese children and adolescents: a systematic review. Int J Pediatr Obes. 2010;5(4):282-304.

10. Buttitta M, lliescu C, Rousseau A, Guerrien A. Quality of life in overweight and obese children and adolescents: a literature review. Qual Life Res. 2014;23(4):1117-39.

11. Braet C, Mervielde I, Vandereycken W. Psychological aspects of childhood obesity: a controlled study in a clinical and nonclinical sample. J Pediatr Psychol. 1997;22(1):59-71.

12. Jansen PW, Mensah FK, Clifford SA, Tiemeier H, Nicholson JM, Wake M. Development of mental health problems and overweight between ages 4 and 11 years: a population-based longitudinal study of Australian children. Acad Pediatr. 2013;13(2):159-67.

13. Vila G, Zipper E, Dabbas M, Bertrand C, Robert JJ, Ricour C, Mouren Siméoni MC. Mental disorders in obese children and adolescents. Psychosomatic Med. 2004;66:387-94.

14. Tanofsky-KraffM, Yanovski SZ, Wilfley DE, Marmarosh C, Morgan CM, Yanovski JA. Eatingdisordered behaviors, body fat, and psychopathology in overweight and normal-weight children. J Cons Clin Psychol. 2004;72(1):53-62.

15. Datar A, Sturm R. Physical education in elementary school and body mass index: evidence from the early childhood longitudinal study. Am J Public Health. 2004;94(9):1501-8.

16. Morgan CM, Tanofsky-Kraff M, Wilfley DE, Yanovski JA. Childhood obesity. Child AdolesC Psychiatric Clin North Am. 2002;11(2):257-78.

17. Lumeng JC, Gannon K, Cabral HJ, Frank DA, Zuckerman B. Association Between Clinically Meaningful Behavior Problems and Overweight in Children. Pediatrics. 2003;112:1138-45.

18. Mustillo S, Worthman C, Erkanli A, Keeler G, Angold A, Costello EJ. Obesity and Psychiatric Disorder: Developmental Trajectories. Pediatrics. 2003;111:851-59.

19. Sherburne Hawkins S, Law C. A review of risk factors for overweight in preschool children: a policy perspective. Int J Pediatr Obes. 2006;1(4):195-209.

20. Santos IS, Barros AJ, Matijasevich A, Domingues MR, Barros FC, Victora CG. Cohort profile: the 2004 Pelotas (Brazil) birth cohort study. Int J Epidemiol. 2011;40(6):1461-68.

21. Achembach TM. Manual for the Child Behavior Checklist/4-18 and profile. Burlington: Department of Psychiatry, University of Vermont; 1991.

22. Bordin IA, Mari JJ, Caeiro MF. Validação da versão brasileira do "Child Behavior Checklist" (CBCL): dados preliminares. Rev ABP-APAL. 1995;17(2):55-66.

23. Rescorla L, Ivanova MY, Achenbach TM, Begovac I, Chahed M, Drugli MB, et al. International epidemiology of child and adolescent psychopathology II: integration and applications of dimensional findings from 44 societies. J Am Acad Child Adolesc Psychiatry. 2012;51(12):1273-83.

24. Garthus-Niegel S, Hagtvet KA, Vollrath ME. A prospective study of weight development and behavior problems in toddlers: the Norwegian Mother and Child Cohort Study. BMC Public Health. 2010;10(1):626-36.

25. Lawlor DA, Mamun AA, O'Callaghan MJ, Bor W, Williams GM, Najman JM. Is being overweight associated with behavioural problems in childhood and adolescence? Findings from the Mater-University study of pregnancy and its outcomes. Arch Dis Child. 2005;90(7):692-97.

26. Dockray S, Susman EJ, Dorn LD. Depression, cortisol reactivity, and obesity in childhood and adolescence. J Adolesc Health. 2009:45(4):344-50. 
27. Bradley RH, Houts $R$, Nader PR, $O^{\prime}$ Brien $M$, Belsky J, Crosnoe $R$. The relationship between body mass index and behaviour in children. J Pediatr. 2008;153(5):629-34.

28. Erickson S, Robinson T, Haydel F, Killen J. Are overweight children unhappy? Arch Pediatr Adolesc Med. 2000;154:931-35.

29. Wei S, Di Santo A. Preschool children's perceptions of overweight peers. J Child Res. 2011;10(1):19-31.

30. Latner JD, Stunkard A. Getting worse: the stigmatization of obese children. Obes Res. 2003;11:452-56.

31. Bauer KW, Yang YW, Austin SB. "How can we stay healthy when you're throwing all of this in front of us?" Findings from focus groups and interviews in middle schools on environmental influences on nutrition and physical activity. Health Educ Behav. 2004;31(1):34-46.

32. Davison KK, Birch LL. Predictors of fat stereotypes among 9-year-old girls and their parents. Obes Res. 2004;12:86-94

33. Cramer P, Steinwert T. Thin is good, fat is bad: how early does it begin? J Appl Dev Psychol. 1998;19(3):429-51

34. Turnbull JD, Heaslip S, McLeod HA. Pre-school children's attitudes to fat and normal male and female stimulus figures. Int J Obes Relat Metab Disord. 2000;24(12):1705-6.

35. Pierce JW, Wardle J. Cause and effect beliefs and self-esteem of overweight children. J Child Psychol Psychiatry. 1997;38(6):645-50.
36. Griffiths LJ, Dezateux C, Hill A. Is obesity associated with emotional and behavioural problems in children? Findings from the Millennium Cohort Study. Int J Pediatr Obes. 2011;6(2-2):e423-32.

37. Cabaj LL, McDonald SW, Tough SC. Early childhood risk and resilience factors for behavioural and emotional problems in middle childhood. BMC Pediatr. 2014;14(1):166-76.

38. Achenbach TM, McConaughy SH, Howell CT. Child/adolescent behavioral and emotional problems: implications of cross informant correlations for situational specificity. Psychol Bull. 1987;101:213-32.

39. Ringoot AP, Tiemeier H, Jaddoe VW, So P, Hofman A, Verhulst FC, et al. Parental depression and child well-being: young children's self-reports helped addressing biases in parent reports. J Clin Epidemiol. 2015;68(8):928-38.

40. Slemming K, Sorensen M, Thomsen PH, Obel C, Henriksen TB, Linnet KM. The association between preschool behavioural problems and internalizing difficulties at age 10-12 years. Eur Child Adolesc Psychiatry. 2010;19:787-95.

41. Hasenboehler K, Munsch S, Meyer AH, Kappler C, Vögele C. Family structure, body mass index, and eating behavior. Int J Eat Dis. 2009;42(4):332-8.

42. Birch LL. Psychological influences on the childhood diet. J Nutrition. 1998;128(2): 407-10.

43. McConley RL, Mrug S, Gilliland MJ, Lowry R, Elliott MN, Schuster MA, et al. Mediators of maternal depression and family structure on child BMI: parenting quality and risk factors for child overweight. Obesity. 2011;19(2):345-52 